\title{
Lumbar Extensor Muscle Size and Isometric Muscle Strength in Women with Symptomatic Lumbar Degenerative Diseases
}

\author{
Yong Gon Seo ${ }^{1}$, Won Hah Park ${ }^{1}$, Chong Suh Lee ${ }^{1}$, Kyung Chung Kang ${ }^{2}$ \\ ${ }^{I}$ Division of Sports Medicine, Department of Orthopedic Surgery, Samsung Medical Center, Sungkyunkwan University School of Medicine, Seoul, Korea \\ ${ }^{2}$ Department of Orthopedic Surgery, Kyung Hee University Hospital, Kyung Hee University School of Medicine, Seoul, Korea
}

Study Design: A cross-sectional design.

Purpose: To determine the characteristics of lumbar extensor muscle (LEM) size and isometric muscle strength and examine their correlations in women with lumbar degenerative diseases (LDDs).

Overview of Literature: Many studies have evaluated the relationship between muscle size and strength, but the results have been controversial.

Methods: Seventy-four female patients (mean age, 66 years) who consecutively underwent posterior lumbar interbody fusion (L1S1) were recruited. The cross-sectional area (CSA) of the back extensor muscles was measured between L1-2 to L5-S1, and the total sum of the CSAs at each disc level was calculated. Back extensor muscle strength was evaluated using a MedX lumbar extension machine. The Oswestry Disability Index (ODI, 0-100) and Visual Analog Scale (VAS, 0-10) of lower back pain were determined.

Results: The mean CSAs of the LEM at each level (L1/2-L5/S1) and the total sum were 34.3, 36.3, 35.1, 31.4, 21.9, and $156.2 \mathrm{~cm} 2$, respectively. The mean isometric strength at each angle (range, $0^{\circ}-72^{\circ}$ ) was $32.5,50.1,72.0,88.7,100.7,112.2$, and $126.2 \mathrm{ft}-\mathrm{lb}$, respectively. The mean ODI and VAS scores were 54.6 and 6.6, and the mean body weight and body mass index (BMI) were $59.9 \mathrm{~kg}$ and $24.9 \mathrm{~kg} / \mathrm{m}^{2}$, respectively. The CSAs of the upper lumbar level (L1-4) and the total sum of the CSAs were associated with isometric strength, which was negatively correlated with patients' age and ODI and positively associated with body weight and BMI, mainly at higher lumbar flexion angles $\left(48^{\circ}-72^{\circ}\right)$.

Conclusions: In women with LDD, LEM sizes of the upper lumbar levels (L1-4) were larger than those of the lower levels (L4-S1) and were positively associated with muscle strength. The upper lumbar levels in patients with LDDs appear to play a compensatory role when degenerative lesions are present in the lower lumbar levels.

Keywords: Back muscles; Muscle strength; Cross-sectional anatomy; Intervertebral disc degeneration

\section{Introduction}

Lumbar extensor muscle size and strength have been considered to play an essential role in patients with lower back pain and functional disability and the planning of rehabilitation modalities [1-3]. There are many studies concerning back muscle degeneration or atrophy and the decrease in back extensor muscle strength in patients with

Received Sep 8, 2017; Revised Oct 27, 2017; Accepted Jan 5, 2018

Corresponding author: Kyung Chung Kang

Department of Orthopedic Surgery, Kyung Hee University Hospital, Kyung Hee University School of Medicine, 23 Kyungheedae-ro, Dongdaemun-gu, Seoul 02447, Korea

Tel: +82-2-958-8346, Fax: +82-2-964-3865, E-mail: futurespine@gmail.com 
lower back pain $[2,4,5]$. Back muscle strength in patients with symptomatic lumbar degenerative disease has also been shown to decrease, particularly in females and older patients [6]. The maintenance of lumbar extensor strength is critical to improving physical function after lumbar fusion in patients with lumbar disc disease because back extensor strength is significantly decreased [7].

Back muscle size is measured in cross-sectional areas (CSAs) and has been previously used as a parameter to evaluate the relationship of muscle strength with variables such as age and weight $[2,4]$. Several studies have reported that the CSA of back muscle is one of the most powerful predictors of back muscle strength $[3,8]$. However, Parkkola et al. [9] reported that the size of back muscles was not related to the maximum extension strength of the trunk. Likewise, there is no consensus regarding the correlation between the CSAs of lumbar muscles and muscle strength, which could be explained by the use of different methods, such as isokinetic or isometric tests, for evaluating lumbar back muscle strength in previous studies $[3,8,9]$.

Various equipment has been developed to correctly quantify the strength of back muscles and to exercise for patients with lower back pain and dysfunction $[2,3,8,9]$. However, controversy remains over objective measurement methods of back muscle strength and correlations between back muscle strength and radiological or clinical parameters [10]. Of the many techniques available, an isometric back muscle strength test is one of the most objective method [11,12]. Keller et al. [8] evaluated back muscle strength using a Roman Chair device with the BieringSorensen test, Hakkinen et al. [13] used a strain-gauge dynamometer, and Roussel et al. [14] tested strength by positioning the equipment in an upright sitting position. The evaluation of lumbar strength using a MedX lumbar extension demonstrated high reliability of repeated measurements of isometric lumbar extension through a range of motion [6]. Few studies have investigated distinctive characteristics of muscle size and strength or their correlation in patients with lumbar degenerative diseases.

This study aims to determine the characteristics of lumbar extensor muscle size and strength and to examine their correlation in women with lumbar degenerative diseases. These results will aid in the planning of rehabilitation of patients with lumbar degenerative disease to specifically improve function and reduce pain.

\section{Materials and Methods}

\section{Study participants}

Between 2007 and 2009, we recruited a final cohort of 74 female patients who failed adequate conservative management for $>6$ months and consecutively underwent posterior lumbar interbody fusion for lumbar degenerative disease. Those who could not adhere to the protocol of lumbar extensor strength measurement and those who underwent simple decompression without instrumentation, a previous lumbar spinal operation, fusion surgery for infection or fracture, and lumbar fusion of four or more segments (for lumbar degenerative kyphosis) were excluded.

All data were prospectively collected before surgery. The mean patient age was 66 years (range, $46-72$ years). The weight of each patient was measured to calculate the body mass index (BMI). The Oswestry Disability Index (ODI; range, 0-100) and pain Visual Analog Scale (VAS; range, $0-10)$ of the lower back were also recorded.

The study population included patients with combined degenerative disc disease and spinal stenosis $(n=42)$, degenerative spondylolisthesis $(n=21)$, spondylolytic spondylolisthesis $(n=8)$, and segmental instability with degenerative disc disease $(n=3)$. The patients underwent surgery at intervertebral disc level from L1-2 to L5-S1 for the fusion of one segment (L4-5, $n=26$ ), two segments (L4-S1, $\mathrm{n}=33$ ), three segments (L3-S1, $\mathrm{n}=10$ ), or four segments $(\mathrm{L} 1-5, \mathrm{n}=5)$. A) This study protocol was approved by the Institutional Review Board of Samsung Medical Center (IRB approval no., SMC 2007-09-033).

\section{Lumbar extensor muscle size}

The CSA of the lumbar extensor muscle including the multifidus and erector spinae muscles was measured at each intervertebral disc level between L1-2 and L5-S1 on an axial T2-weighted magnetic resonance imaging (MRI), and the total sum of all CSAs from L1-2 to L5-S1 was calculated $[15,16]$. The sizes of the back extensor muscle were measured on each axial MRI by constructing polygon points around the outer margin of the extensor muscle in the picture archiving and communication system. The measurers attempted to maintain the same distance between each point of the polygon (Fig. 1). To minimize inter and intraobserver errors, two orthopedic surgeons evaluated the digitized radiographs twice using MRIs with 

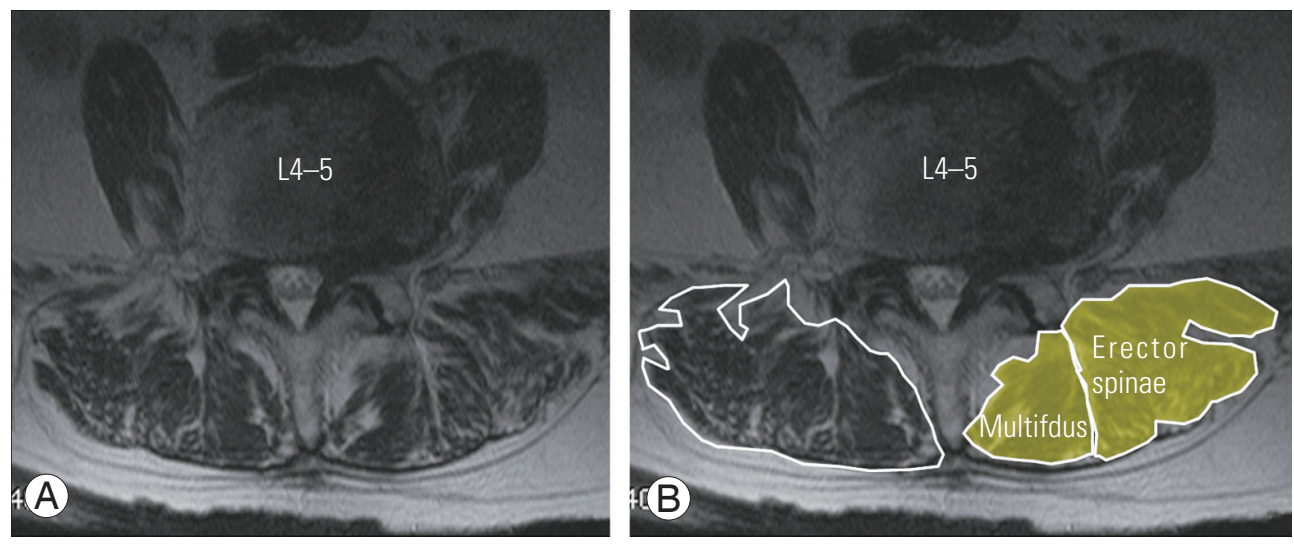

Fig. 1. Cross-sectional areas of the back extensor muscle were measured at each intervertebral disc level between L1-2 and L5-S1. (A) T2-weighted axial section of an magnetic resonance imaging of a 70-year-old woman at the L4-5 intervertebral disc space. (B) The back extensor muscles included the multifidus and erector spinae muscles.
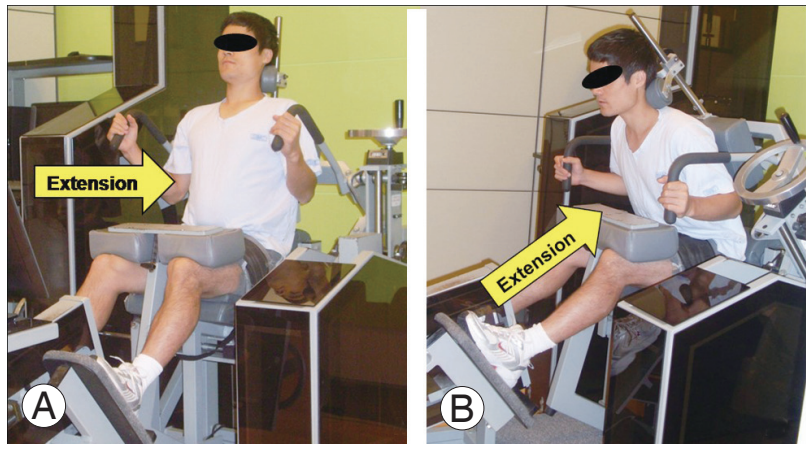

Fig. 2. Back muscle strength was evaluated by measuring the isometric strength using a MedX lumbar extension machine. The maximal voluntary isometric strength of the lumbar extensor muscles at $0^{\circ}$ (A) $-72^{\circ}$ (B) of lumbar flexion was measured.

$2 \times$ magnification. The inter- and intraobserver intraclass correlation coefficients were assessed for the CSA of the back extensor muscles ( 0.816 and 0.863 , respectively).

\section{Lumbar extensor muscle strength}

Prior to surgery, the maximal voluntary isometric strength of the lumbar extensor muscles (ft-lb) was measured using a MedX lumbar extension machine (MedX, Ocala, FL, USA) at $0^{\circ}, 12^{\circ}, 24^{\circ}, 36^{\circ}, 48^{\circ}, 60^{\circ}$, and $72^{\circ}$ of lumbar flexion. All subjects were thoroughly instructed on the method for accurate testing and were asked to perform warm-up exercises 15 minutes beforehand. The patients were positioned according to the established protocol and asked to increase the lumbar extension torque over 3 seconds. After reaching the maximum torque, they were asked to slowly decrease the torque for 3 seconds. During contractions, concurrent visual feedback was provided on a video display screen interfaced with the machine, and the patients were verbally encouraged to exert the maximum effort, even if they had a severe dysfunction or back pain (Fig. 2) [11,17]. A 10-second rest period was allowed between angle conditions. All subjects completed the seven trials of lumbar isometric extension strength tests, and the average test strength was used in this study. All patients were assessed by one examiner who was blinded to other results.

\section{Oswestry Disability Index/Visual Analog Scale and body composition parameters}

ODI (0-100) and VAS (0-10) scores of lower back pain were recorded. Body composition parameters (body weight and BMI) were obtained using an InBody ver. 4.0 body composition analyzer (Biospace, Seoul, Korea).

\section{Statistical analysis}

A professional medical statistical consultant performed the statistical analyses using SAS ver. 9.1 (SAS Institute Inc., Cary, NC, USA). Correlations between isometric back muscle strength and radiographic (CSA of back muscle) or clinical (age, body weight, BMI, ODI, and VAS) parameters were analyzed using Pearson's product moment correlation coefficient and Spearman's rank correlation coefficient (rho), and Bonferroni's corrections 
were conducted on each $p$-value. A $p$-value of $<0.05$ was considered significant.

\section{Results}

\section{Isometric back muscle strength versus cross-sectional area of back muscle}

The mean isometric strengths at each angle $\left(0^{\circ}, 12^{\circ}\right.$, $24^{\circ}, 36^{\circ}, 48^{\circ}, 60^{\circ}$, and $72^{\circ}$ ) were $32.5 \pm 21.9,50.1 \pm 29.2$,

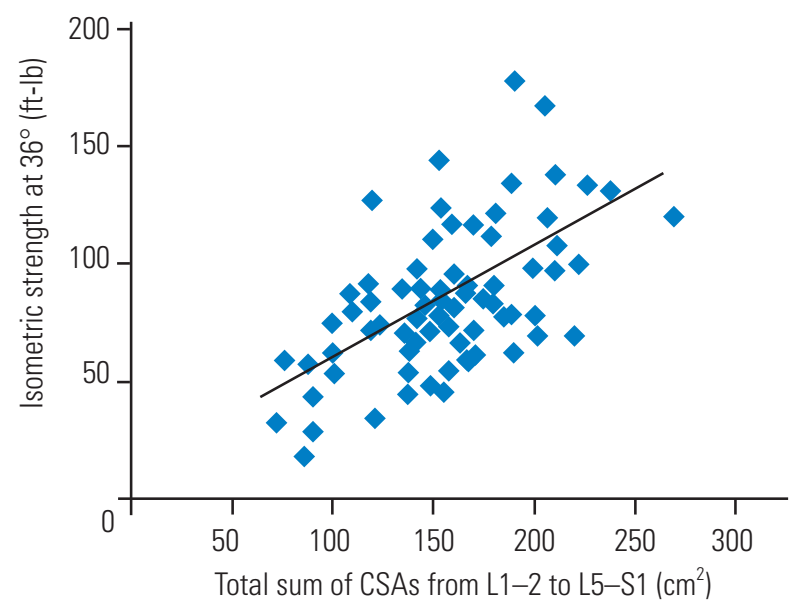

Fig. 3. Isometric back muscle strength at all lumbar flexion angles $\left(0^{\circ}-72^{\circ}\right)$ was associated with the total sum of CSAs of the back extensor muscle $(p<0.05)$. Among them, isometric strength at $36^{\circ}$ showed the most significant correlation with the total sum of CSAs $(p=0.001$, correlation coefficient $[r]=0.590)$. CSA, cross-sectional area.
$72.0 \pm 33.0,88.7 \pm 37.5,100.7 \pm 41.0,112.2 \pm 43.6$, and $126.2 \pm 47.2 \mathrm{ft}-\mathrm{lb}$. The mean CSAs of the back extensor muscle at each intervertebral disc level were $34.3 \pm 8.2$

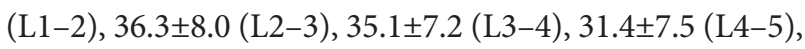
and $21.9 \pm 6.9$ (L5-S1) $\mathrm{cm}^{2}$ and the total sum of all CSAs (L1-S1) was $156.2 \pm 34.7 \mathrm{~cm}^{2}$.

The isometric back muscle strengths at all lumbar flexion angles were significantly associated with the total sum of the CSAs of the back extensor muscles $(p<0.05)$ (Fig. $3)$. The isometric strengths were mainly associated with the CSAs of the upper lumbar level back extensor muscles (from L1-2 to L3-4), but scarcely with the CSAs of the lower lumbar level back extensor muscles (L4-5 and L5S1). Notably, isometric strengths were strongly correlated with the CSA at L1-2 intervertebral disc level, and no significant correlation with the CSA at L5-S1 intervertebral disc level was observed (Table 1).

\section{Correlation between isometric back muscle strength versus age, Oswestry Disability Index/Visual Analog Scale, and body composition parameters}

The mean ODI and pain VAS scores for the lower back were $54.7 \pm 12.4$ and $6.6 \pm 2.5$, respectively. The mean body weight and BMI of the patients were $59.9 \pm 11.4 \mathrm{~kg}$ and $24.9 \pm 3.74 \mathrm{~kg} / \mathrm{m}^{2}$, respectively.

The isometric back muscle strengths at angles $12^{\circ}-60^{\circ}$ were negatively associated with patients' age. Body weight and BMI were associated with isometric strength at all

Table 1. Correlations between isometric back muscle strengths at each lumbar flexion angle (from $0^{\circ}$ to $72^{\circ}$ ) and CSA of back muscles at each intervertebral disc level (from L1-2 to L5-S1) and total sum of CSAs

\begin{tabular}{|c|c|c|c|c|c|c|}
\hline \multirow{2}{*}{ Variable } & \multicolumn{6}{|c|}{ CSA of back muscles (intervertebral disc level) } \\
\hline & $\mathrm{L} 1-2$ & $\mathrm{~L} 2-3$ & L3-4 & $\mathrm{L} 4-5$ & L5-S1 & Total sum of L1-S1 \\
\hline \multicolumn{7}{|c|}{ Isometric back muscle strength (angle of lumbar flexion) } \\
\hline $0^{\circ}$ & $0.560^{* *}$ & $0.362^{*}$ & 0.319 & 0.168 & 0.179 & $0.397^{*}$ \\
\hline $12^{\circ}$ & $0.584^{* *}$ & 0.248 & 0.285 & 0.101 & 0.195 & $0.379^{*}$ \\
\hline $24^{\circ}$ & $0.683^{* * *}$ & $0.538^{* *}$ & $0.490^{* *}$ & 0.274 & 0.231 & $0.523^{* *}$ \\
\hline $36^{\circ}$ & $0.726^{* * *}$ & $0.601^{* * *}$ & $0.565^{* *}$ & $0.339 *$ & 0.289 & $0.590^{* *}$ \\
\hline $48^{\circ}$ & $0.712^{* * *}$ & $0.586^{* * *}$ & $0.525^{* *}$ & 0.297 & 0.242 & $0.557^{* *}$ \\
\hline $60^{\circ}$ & $0.685^{* * *}$ & $0.531 * *$ & $0.471^{* *}$ & 0.245 & 0.181 & $0.513^{* *}$ \\
\hline $72^{\circ}$ & $0.683^{* * *}$ & $0.540^{* *}$ & $0.480^{* *}$ & 0.267 & 0.201 & $0.516^{* *}$ \\
\hline
\end{tabular}

Values are presented as correlation coefficients $(r)$ by Pearson's product moment correlation and Spearman's rank correlation rho. CSA, cross-sectional area.

" $p<0.05,{ }^{* *} p<0.01,{ }^{* * *} p<0.001$. 
Table 2. Correlations between isometric back muscle strengths at each lumbar flexion angle $\left(0^{\circ}, 12^{\circ}, 24^{\circ}, 36^{\circ}, 48^{\circ}, 60^{\circ}\right.$, and $\left.72^{\circ}\right)$ and age, $0 \mathrm{DI} /$ VAS, or body composition parameters

\begin{tabular}{|c|c|c|c|c|c|}
\hline Variable & Age & Body weight & Body mass index & ODI & VAS \\
\hline \multicolumn{6}{|c|}{ Isometric back muscle strength (angle of lumbar flexion) } \\
\hline $0^{\circ}$ & -0.274 & $0.398^{*}$ & $0.355^{*}$ & $-0.343^{*}$ & -0.310 \\
\hline $12^{\circ}$ & $-0.425^{* *}$ & $0.384^{*}$ & $0.396^{*}$ & $-0.321^{*}$ & -0.283 \\
\hline $24^{\circ}$ & $-0.451^{* *}$ & $0.529^{* * *}$ & $0.496^{* * *}$ & $-0.314^{*}$ & -0.249 \\
\hline $36^{\circ}$ & $-0.442^{* *}$ & $0.580^{* * *}$ & $0.546^{* * *}$ & $-0.306^{*}$ & -0.234 \\
\hline $48^{\circ}$ & $-0.418^{* *}$ & $0.632^{* * *}$ & $0.589^{* * *}$ & -0.172 & -0.176 \\
\hline $60^{\circ}$ & $-0.348^{*}$ & $0.661^{* * *}$ & $0.609 * * *$ & $-0.344^{*}$ & -0.188 \\
\hline $72^{\circ}$ & -0.297 & $0.704^{* * *}$ & $0.658^{* * *}$ & $-0.313^{*}$ & -0.261 \\
\hline
\end{tabular}

Values are presented as correlation coefficients $(r)$ by Pearson's product moment correlation and Spearman's rank correlation rho. ODI, Oswestry Disability Index; VAS, Visual Analog Scale.

$p<0.05,{ }^{* *} p<0.01,{ }^{* * *} p<0.001$

lumbar flexion angles, demonstrating particularly strong correlations with the isometric strength at higher lumbar flexion angles $\left(48^{\circ}-72^{\circ}\right)$. Isometric strengths at most of the lumbar flexion angles showed significant but weak negative correlations with ODI (correlation coefficients $<0.4)$. VAS scores showed only a tendency toward negative correlation with isometric strength at lower angles $\left(0^{\circ}, p=0.055 ; 12^{\circ}, p=0.077\right)$, but this was not statistically significant (Table 2).

Additionally, correlation analyses between ODI and other variables revealed that ODI was significantly associated with age ( $p=0.031, r=0.330)$ and isometric strength $(p=0.050, r=-0.306)$ and showed a tendency toward positive correlation with body weight ( $p=0.064, r=-0.285$ ).

\section{Discussion}

In this study, isometric strength was associated with the total sum of the CSAs of the back extensor muscles, mainly with the CSAs of the upper lumbar back muscles from L1-2 to L3-4. The isometric strength at most of the angles of lumbar flexion showed significant negative correlations with ODI score, but not with VAS score. Also, patient age was negatively associated with isometric strength at lumbar flexion angles of $12^{\circ}-60^{\circ}$. Body weight and BMI were associated with isometric strength, especially at higher angles $\left(48^{\circ}-72^{\circ}\right)$.

Many studies have reported the association of chronic lower back pain and decreased back muscle strength and size [2-4]. The CSA measurement was considered the most easily accessible method without requiring a special- ized program or device [18]. However, the measurement of muscle CSAs had some potential bias, such as infiltrating fat components, inhomogeneous muscle contents, and irregular margins between muscle and fat. Previous study results of correlations between muscle strength and size are controversial. Parkkola et al. [9] verified that isometric back muscle strength, determined using specialized equipment, was not related with the measured CSA of the back muscle (L4-5). Another study [3] also demonstrated a lack of correlation between the CSA of back extensors and the extensor strength. However, Peltonen et al. [19] found that an increased CSA of back extensors was correlated with increased back muscle strength. We suggest that the discrepancy in the results of previous studies may be due to the measuring technique (computed tomography $[8,20]$ versus MRI $[16,21]$, or isometric $[6,7,14]$ versus isokinetic [4]) or the measuring site (L3 versus L4 versus L5) as well as the fatty infiltration ratio and muscle fiber elasticity.

Our present study revealed an association between back extensor strength and CSA at the upper intervertebral disc levels from L1-2 to L3-4, but not at the lower levels from L4-5 to L5-S1. In general, the muscle at the L1-2 level was not considered clinically significant because most of the lumbar degenerative diseases occurred at the lower lumbar levels. Previous studies have proposed that the back muscle at the upper lumbar level plays a compensatory role for the atrophied back muscle at the lower lumbar level $[22,23]$. Therefore, to correctly predict the back muscle strength or remaining functional potential of patients with severe lumbar degenerative disease, we recommend that evaluation considering the testing position 
of the upper lumbar muscles is necessary.

In our study, isometric back muscle strength showed a significant inverse correlation with the ODI score. Although the correlation was weak $(r<-0.4)$, we thought this result clinically important because few studies have shown a direct association between back muscle strength and patient dysfunction [24,25], and this could be used as basic data in back muscle strengthening exercise programs for patients with lumbar degenerative disease. Furthermore, additional studies using other function-specific assessments, such as the Roland-Morris Disability Questionnaire and 36-item Short-Form Health Survey, would be helpful to elucidate the relationship between back muscle strength and dysfunction. On the other hand, the VAS score did not demonstrate a significant correlation with isometric strength and only showed a tendency toward negative correlations with isometric strength at lower angles $\left(0^{\circ}-12^{\circ}\right)$. A lower angle $\left(0^{\circ}-12^{\circ}\right)$ in the MedX isometric muscle test means a maximally extended trunk position. Although our results contradict previous reports that showed the association between lower back pain and decreased muscle strength $[1,26]$, this result could be an important clinical finding because most patients with degenerative disc disease complain of severe back pain when the trunk is in an extended position [27]. Based on the finding that strong back extensor muscles at lower angles are related to decreased lower back pain, we propose that isometric back extensor strength in the extended position could be an important clinical factor to evaluate the correlation between back muscle strength and back pain severity.

Isometric strength at higher lumbar flexion angles was associated with body weight and BMI. This result is consistent with previous studies $[10,28]$ that demonstrated age and BMI as influencing factors in females. In the MedX isometric muscle test, the back extensor strength tends to gradually decrease as the trunk extension angle increases [6]. The absolute back extensor muscle strength can be obtained at a flexion angle of $72^{\circ}$ because the optimal muscle length, explained as the length-force relationship, is observed at $72^{\circ}$ flexion angle to evaluate the maximal muscle force, and it gradually decreases as the trunk extension angle increases [29]. Based on previous studies $[28,29]$, we propose that the maximal muscle force could be demonstrated in patients with a higher BMI because the absolute back muscle strength of our results did not take into account the body weight of each patient [28]. We recommended that the relative results (results $/ \mathrm{kg}$ ), taking into account the body weight, should be evaluated for measuring the function of back extensor muscles in these patients.

This study had some limitations. First, we did not examine a control group of healthy individuals. The results of comparison with a control group could aid the establishment of standards in back muscle strengthening exercises. Second, we only examined the back extensor muscles. Because lumbar spine motion is multiplanar, the evaluation of other paraspinal muscles including abdominal muscles, the psoas, quadratus lumborum, or gluteal muscles is necessary for the thorough investigation of back muscles. In this study, however, we focused on the extensor muscle strength and size and examined the correlation between extensor muscles and clinical parameters. Lastly, it was difficult to precisely evaluate the back muscle strength of the patients due to their back pain. To minimize this bias, one specialist at our sports medicine center assessed all patients' muscle strengths and continually encouraged them to exert maximal effort during the testing. The patients who could not correctly perform the protocol of strength measurement were excluded.

\section{Conclusions}

In female patients with lumbar degenerative disease, isometric strength was positively associated with the CSA of back extensor muscles, especially at the upper lumbar level (L1-4), and body weight/BMI and negatively associated with age and ODI score. The back extensor strength at the extended position $\left(0^{\circ}-12^{\circ}\right)$ and upper lumbar level (L1-4) is an important parameter to consider when evaluating muscle function and back pain in these patients. Our findings will be helpful in evaluating back extensor function and prescribing optimal exercise intervention.

\section{Conflict of Interest}

No potential conflict of interest relevant to this article was reported.

\section{Author Contributions}

Conception and design: YGS, KCK, WHP; acquisition of data: YGS, KCK; analysis and interpretation of data: YGS, WHP, CSL, KCK; and statistical analysis: KCK, YGS. 


\section{References}

1. Thorstensson A, Arvidson A. Trunk muscle strength and low back pain. Scand J Rehabil Med 1982;14:6975.

2. Cooper RG, St Clair Forbes W, Jayson MI. Radiographic demonstration of paraspinal muscle wasting in patients with chronic low back pain. Br J Rheumatol 1992;31:389-94.

3. Lee HJ, Lim WH, Park JW, et al. The relationship between cross sectional area and strength of back muscles in patients with chronic low back pain. Ann Rehabil Med 2012;36:173-81.

4. Mayer TG, Vanharanta H, Gatchel RJ, et al. Comparison of CT scan muscle measurements and isokinetic trunk strength in postoperative patients. Spine (Phila Pa 1976) 1989;14:33-6.

5. Sihvonen T, Herno A, Paljarvi L, Airaksinen O, Partanen J, Tapaninaho A. Local denervation atrophy of paraspinal muscles in postoperative failed back syndrome. Spine (Phila Pa 1976) 1993;18:575-81.

6. Park WH, Lee CS, Kang KC, Seo YG. Characteristics of back muscle strength in patients with scheduled for lumbar fusion surgery due to symptomatic lumbar degenerative diseases. Asian Spine J 2014;8:65966.

7. Seo YG, Lee CS, Kang KC, Park WH. Change of lumbar isometric extensor strengths after posterior lumbar interbody fusion in patients with lumbar degenerative disease. J Korean Soc Spine Surg 2014;21:1606.

8. Keller A, Johansen JG, Hellesnes J, Brox JI. Predictors of isokinetic back muscle strength in patients with low back pain. Spine (Phila Pa 1976) 1999;24:275-80.

9. Parkkola R, Rytokoski U, Kormano M. Magnetic resonance imaging of the discs and trunk muscles in patients with chronic low back pain and healthy control subjects. Spine (Phila Pa 1976) 1993;18:830-6.

10. Mayer TG, Gatchel RJ, Kishino N, et al. Objective assessment of spine function following industrial injury: a prospective study with comparison group and one-year follow-up. Spine (Phila Pa 1976) 1985;10:482-93.

11. Pollock ML, Leggett SH, Graves JE, Jones A, Fulton $\mathrm{M}$, Cirulli J. Effect of resistance training on lumbar extension strength. Am J Sports Med 1989;17:624-9.

12. Udermann BE, Mayer JM, Graves JE, Murray SR.
Quantitative assessment of lumbar paraspinal muscle endurance. J Athl Train 2003;38:259-62.

13. Hakkinen A, Ylinen J, Kautiainen H, et al. Pain, trunk muscle strength, spine mobility and disability following lumbar disc surgery. J Rehabil Med 2003;35:236-40.

14. Roussel NA, Truijen S, De Kerf I, Lambeets D, Nijs J, Stassijns G. Reliability of the assessment of lumbar range of motion and maximal isometric strength in patients with chronic low back pain. Arch Phys Med Rehabil 2008;89:788-91.

15. Kang CH, Shin MJ, Kim SM, Lee SH, Lee CS. MRI of paraspinal muscles in lumbar degenerative kyphosis patients and control patients with chronic low back pain. Clin Radiol 2007;62:479-86.

16. Hides JA, Belavy DL, Stanton W, et al. Magnetic resonance imaging assessment of trunk muscles during prolonged bed rest. Spine (Phila Pa 1976) 2007;32:1687-92.

17. Graves JE, Webb DC, Pollock ML, et al. Pelvic stabilization during resistance training: its effect on the development of lumbar extension strength. Arch Phys Med Rehabil 1994;75:210-5.

18. Lee SW, Chan CK, Lam TS, et al. Relationship between low back pain and lumbar multifidus size at different postures. Spine (Phila Pa 1976) 2006;31:2258-62.

19. Peltonen JE, Taimela S, Erkintalo M, Salminen JJ, Oksanen A, Kujala UM. Back extensor and psoas muscle cross-sectional area, prior physical training, and trunk muscle strength: a longitudinal study in adolescent girls. Eur J Appl Physiol Occup Physiol 1998;77:66-71.

20. Lee SH, Park SW, Kim YB, Nam TK, Lee YS. The fatty degeneration of lumbar paraspinal muscles on computed tomography scan according to age and disc level. Spine J 2017;17:81-7.

21. Mayer JM, Graves JE, Clark BC, Formikell M, PloutzSnyder LL. The use of magnetic resonance imaging to evaluate lumbar muscle activity during trunk extension exercise at varying intensities. Spine (Phila $\mathrm{Pa}$ 1976) 2005;30:2556-63.

22. Abbas J, Slon V, May H, Peled N, Hershkovitz I, Hamoud K. Paraspinal muscles density: a marker for degenerative lumbar spinal stenosis? BMC Musculoskelet Disord 2016;17:422.

23. Leinonen V, Maatta S, Taimela S, et al. Paraspinal 
muscle denervation, paradoxically good lumbar endurance, and an abnormal flexion-extension cycle in lumbar spinal stenosis. Spine (Phila Pa 1976) 2003;28:324-31.

24. Imagama S, Matsuyama Y, Hasegawa Y, et al. Back muscle strength and spinal mobility are predictors of quality of life in middle-aged and elderly males. Eur Spine J 2011;20:954-61.

25. Aytar A, Pekyavas NO, Ergun N, Karatas M. Is there a relationship between core stability, balance and strength in amputee soccer players?: a pilot study. Prosthet Orthot Int 2012;36:332-8.

26. Bayramoglu M, Akman MN, Kilinc S, Cetin N, Yavuz $\mathrm{N}$, Ozker R. Isokinetic measurement of trunk muscle strength in women with chronic low-back pain. Am J Phys Med Rehabil 2001;80:650-5.
27. Miyakoshi N, Hongo M, Kasukawa Y, Ishikawa Y, Shimada Y. Prevalence, spinal alignment, and mobility of lumbar spinal stenosis with or without chronic low back pain: a community-dwelling study. Pain Res Treat 2011;2011:340629.

28. Kim JW, Seo DI, Swearingin B, So WY. Association between obesity and various parameters of physical fitness in Korean students. Obes Res Clin Pract 2013;7:e67-74.

29. Kaufman KR, An KN, Chao EY. Incorporation of muscle architecture into the muscle length-tension relationship. J Biomech 1989;22:943-8. 